\title{
A BLOWUP ANALYSIS OF THE MEAN FIELD EQUATION FOR ARBITRARILY SIGNED VORTICES
}

\author{
HIROSHI OHTSUKA \\ Department of Applied Physics, Faculty of Engineering \\ University of Miyazaki, Japan \\ E-mail: ohtsuka@cc.miyazaki-u.ac.jp \\ TAKASHI SUZUKI \\ Division of Mathematical Science, Department of System Innovation \\ Graduate School of Engineering Science, Osaka University, Japan \\ E-mail: suzuki@sigmath.es.osaka-u.ac.jp
}

\begin{abstract}
We study the noncompact solution sequences to the mean field equation for arbitrarily signed vortices and observe the quantization of the mass of concentration, using the rescaling argument.
\end{abstract}

1. Introduction. We continue the study [34] on the noncompact solution sequences to the mean field equation for arbitrarily signed vortices on a two-dimensional compact orientable Riemannian manifold $(M, g)$ without boundary:

$$
\begin{aligned}
& -\Delta_{g} v=\lambda_{1}\left(\frac{e^{v}}{\int_{M} e^{v} d v_{g}}-\frac{1}{|M|}\right)-\lambda_{2}\left(\frac{e^{-v}}{\int_{M} e^{-v} d v_{g}}-\frac{1}{|M|}\right), \\
& \int_{M} v d v_{g}=0,
\end{aligned}
$$

where $\Delta_{g}, d v_{g}$, and $|M|$ are the Laplace-Beltrami operator, the volume form, and the volume of $M$, respectively, and $\lambda_{1}, \lambda_{2}$ are nonnegative constants.

This equation is derived by Joyce and Montgomery [20] and Pointin and Lundgren [35] from different statistical arguments for describing the mean field of the equilibrium turbulence with arbitrarily signed vortices, see also [28, 12, 25, 30]. Here, these vortices are composed of positive and negative intensities with the same absolute value, and $v$ and

2000 Mathematics Subject Classification: 35Q, 35J60, 35B40.

Key words and phrases: mean field equation, Liouville system, blow-up analysis, symmetrization, rescaling.

The paper is in final form and no version of it will be published elsewhere. 
$\lambda_{1} / \lambda_{2}$ are associated with the stream function of the fluid and the ratio of the numbers of the signed vortices, respectively.

The equation (1) is the Euler-Lagrange equation of the functional

$$
J_{\lambda_{1}, \lambda_{2}}(v)=\frac{1}{2} \int_{M}\left|\nabla_{g} v\right|^{2} d v_{g}-\lambda_{1} \log \int_{M} e^{v} d v_{g}-\lambda_{2} \log \int_{M} e^{-v} d v_{g}
$$

defined on

$$
E=\left\{w \in H^{1}(M) \mid \int_{M} w d v_{g}=0\right\},
$$

which forms a Hilbert space with the inner product $\langle u, v\rangle=\int_{M} \nabla_{g} u \cdot \nabla_{g} v d v_{g}$. When $\left(\lambda_{1}, \lambda_{2}\right)=(\lambda, 0)$ or $\left(\lambda_{1}, \lambda_{2}\right)=(0, \lambda)$, this $J_{\lambda_{1}, \lambda_{2}}$ is reduced to

$$
I_{\lambda}(v)=\frac{1}{2} \int_{M}\left|\nabla_{g} v\right|^{2} d v_{g}-\lambda \log \int_{M} e^{v} d v_{g}
$$

and it is associated with the Trudinger-Moser inequality [16] given by

$$
\begin{array}{ll}
\inf _{v \in E} I_{\lambda}(v)>-\infty & \text { if } \lambda \in[0,8 \pi], \\
\inf _{v \in E} I_{\lambda}(v)=-\infty & \text { if } \lambda>8 \pi .
\end{array}
$$

We have

$$
J_{\lambda_{1}, \lambda_{2}}(v)=\frac{1}{2}\left(1-\frac{\lambda_{1}}{8 \pi}-\frac{\lambda_{2}}{8 \pi}\right)\|v\|_{E}^{2}+\frac{\lambda_{1}}{8 \pi} I_{8 \pi}(v)+\frac{\lambda_{2}}{8 \pi} I_{8 \pi}(-v),
$$

and therefore,

$$
\inf _{v \in E} J_{\lambda_{1}, \lambda_{2}}(v)>-\infty \quad \text { if } 1-\frac{\lambda_{1}}{8 \pi}-\frac{\lambda_{2}}{8 \pi} \geq 0
$$

In our previous work [34] we improve this trivial inequality to the following optimal one:

TheOREM 1.1.

$$
\inf _{v \in E} J_{\lambda_{1}, \lambda_{2}}(v)>-\infty \quad \text { if }\left(\lambda_{1}, \lambda_{2}\right) \in[0,8 \pi] \times[0,8 \pi],
$$

and in particular $J_{\lambda_{1}, \lambda_{2}}$ has a global minimizer on $E$ if $0 \leq \lambda_{1}, \lambda_{2}<8 \pi$ and

$$
\inf _{v \in E} J_{\lambda_{1}, \lambda_{2}}(v)=-\infty \quad \text { if } \lambda_{1}>8 \pi \text {, or } \lambda_{2}>8 \pi \text {. }
$$

We note that similar results for $H_{0}^{1}(\Omega)$ on a bounded domain $\Omega \subset \mathbb{R}^{2}$ follow from the above theorem by a simple extension argument, see [34].

Although Shafrir and Wolansky [37] obtained a related result that leads to (2), we proved the above result by a completely different method. We developed blow-up analysis for the solution sequence to (1), and apply the argument of Jost and Wang [18] concerning $S U(3)$ Toda system. The purpose of this paper is to develop further the blow-up analysis and clarify the possible singular limits of the solution sequence to (1) to some extent.

First, we recall the following result on the blow-up analysis from [34]:

THEOREM 1.2. Let $\left\{\lambda_{1, n}\right\}$ and $\left\{\lambda_{2, n}\right\}$ be sequences of nonnegative constants satisfying

$$
\lambda_{i, n} \rightarrow \lambda_{i}(\geq 0) \quad \text { as } n \rightarrow \infty \text { for } i=1,2,
$$

and $\left\{v_{n}\right\} \subset E$ be a sequence of solutions to (1) corresponding to $\left(\lambda_{1, n}, \lambda_{2, n}\right)$. Then, up to a subsequence, the following alternatives hold: 
(1) (compactness) There exist $v \in E$ and a subsequence of $\left\{v_{n}\right\}$ (denoted by the same symbol, also hereafter) such that

$$
v_{n} \rightarrow v \text { in } E,
$$

where this $v$ is a solution to (1) for those $\lambda_{1}$ and $\lambda_{2}$.

(2) (one-sided concentration) Let $\mathcal{S}_{1}$ and $\mathcal{S}_{2}$ be the blow-up set of (this subsequence of) $\left\{v_{n}\right\}$ and $\left\{-v_{n}\right\}$, respectively, that is,

$$
\begin{aligned}
& \mathcal{S}_{1}=\left\{x \in M \mid \exists x_{n} \rightarrow x \text { s.t. } v_{n}\left(x_{n}\right) \rightarrow+\infty\right\}, \\
& \mathcal{S}_{2}=\left\{x \in M \mid \exists x_{n} \rightarrow x \text { s.t. } v_{n}\left(x_{n}\right) \rightarrow-\infty\right\} .
\end{aligned}
$$

Then there exists $i \in\{1,2\}$ such that $\mathcal{S}_{i} \neq \emptyset$ and $\mathcal{S}_{j}=\emptyset$ for $j \in\{1,2\} \backslash\{i\}$. Moreover, put

$$
\mu_{1, n} \equiv \lambda_{1, n} \frac{e^{v_{n}}}{\int_{M} e^{v_{n}} d v_{g}}, \quad \mu_{2, n} \equiv \lambda_{2, n} \frac{e^{-v_{n}}}{\int_{M} e^{-v_{n}} d v_{g}},
$$

and identify them with $\mu_{k, n} d v_{g}(k=1,2)$ in the space of measures $\mathcal{M}(M)=$ $C(M)^{*}$. Then

$$
\mu_{i, n} \rightarrow \mu_{i}=\sum_{x_{0} \in \mathcal{S}_{i}} 8 \pi \delta_{x_{0}} \quad \text { weakly } * \text { in } \mathcal{M}(M)
$$

and

$$
\mu_{i, n} \rightarrow 0 \quad \text { in } L^{\infty}(\omega)
$$

for every $\omega \Subset M \backslash \mathcal{S}_{i}$. On the other hand, there exists $u_{j} \in E$ and a subsequence of $\left\{u_{j, n}\right\}$ such that

$$
u_{j, n} \rightarrow u_{j} \quad \text { in } E,
$$

where this $u_{j}$ is a solution to

$$
-\Delta_{g} v=\lambda\left(\frac{K(x) e^{v}}{\int_{M} K(x) e^{v} d v_{g}}-\frac{1}{|M|}\right), \quad \int_{M} v d v_{g}=0,
$$

with $K(x)=e^{-\sum_{x_{0} \in \mathcal{S}_{i}} 8 \pi G\left(x, x_{0}\right)}$. Here $G=G(x, y)$ indicates the Green function of $-\Delta_{g}$, that is,

$$
-\Delta_{g} G(\cdot, y)=\delta_{x}-\frac{1}{|M|} \quad \text { in } M, \quad \int_{M} G(\cdot, y) d v_{g}=0 .
$$

(3) (concentration) For each $i=1,2$, we have $\mathcal{S}_{i} \neq \emptyset$ and there exists a positive constant

$$
m_{i}\left(x_{0}\right) \geq 4 \pi \quad \text { for each } x_{0} \in \mathcal{S}_{i} .
$$

We have, furthermore, a nonnegative function

$$
r_{i}(x) \in L^{1}(M) \cap L_{\mathrm{loc}}^{\infty}\left(M \backslash \mathcal{S}_{i}\right)
$$

such that

$$
\mu_{i, n} \rightarrow r_{n}+\sum_{x_{0} \in \mathcal{S}_{i}} m_{i}\left(x_{0}\right) \delta_{x_{0}} \quad \text { weakly } * \text { in } \mathcal{M}(M)
$$

and

$$
\mu_{i, n} \rightarrow r_{i} \quad \text { in } L^{p}(\omega)
$$

for every $p \in[1, \infty)$ and every $\omega \Subset M \backslash \mathcal{S}_{i}$. Finally, the following facts hold: 
3-i) If there exists $x_{0} \in \mathcal{S}_{i} \backslash \mathcal{S}_{j}$ for $i \neq j$, then we have $m_{i}\left(x_{0}\right)=8 \pi$ and $r_{i} \equiv 0$.

3-ii) For every $x_{0} \in \mathcal{S}_{1} \cap \mathcal{S}_{2}$, we have

$$
\left(m_{1}\left(x_{0}\right)-m_{2}\left(x_{0}\right)\right)^{2}=8 \pi\left(m_{1}\left(x_{0}\right)+m_{2}\left(x_{0}\right)\right) .
$$

Moreover, if $\mathcal{S}_{i} \subset \mathcal{S}_{j}$ and there exists $x_{0} \in \mathcal{S}_{i}$ satisfying

$$
m_{i}\left(x_{0}\right)-m_{j}\left(x_{0}\right)>4 \pi
$$

then $r_{i} \equiv 0$, see Figure 1 .

In this paper, we improve the minimum mass (5) as follows:

TheOREM 1.3 (Main Result). In the conclusion of Theorem 1.2, (5) is improved as follows, see Figure 1:

$$
m_{i}\left(x_{0}\right) \geq 8 \pi \quad \text { for each } x_{0} \in \mathcal{S}_{i} \text {. }
$$

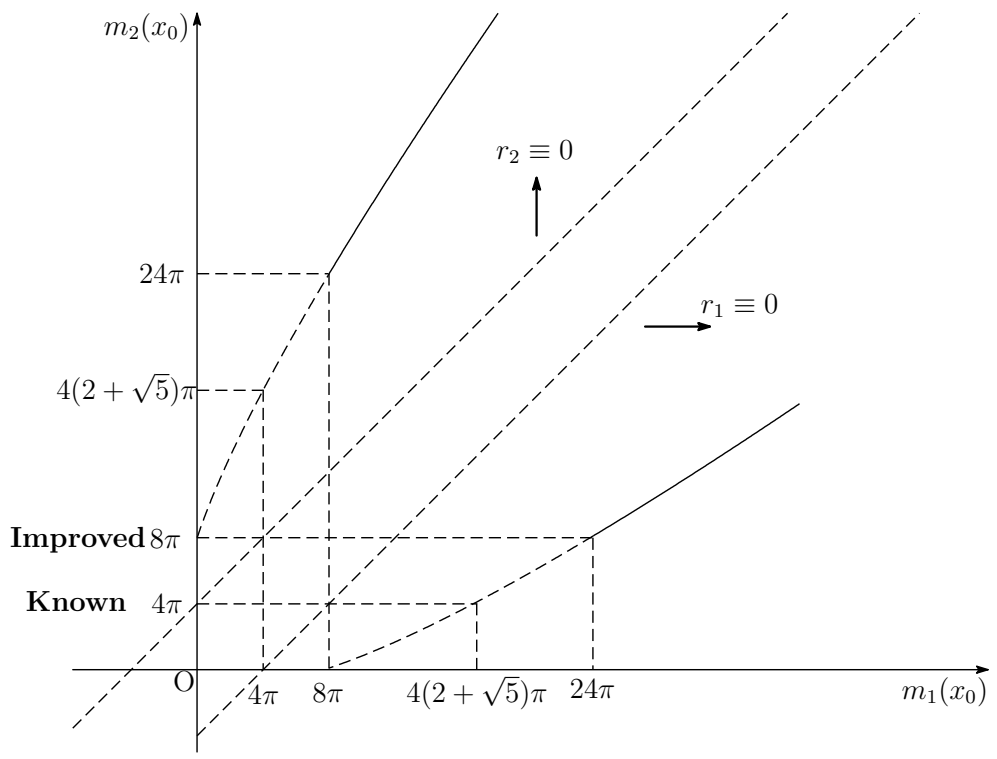

Fig. 1. The mass of concentration at $x_{0} \in \mathcal{S}_{1} \cap \mathcal{S}_{2}$

The conclusion follows from Theorem 1.23 -i) when $x_{0} \in \mathcal{S}_{i} \backslash \mathcal{S}_{j}$ for some $i \neq j$. Thus we only consider the case $x_{0} \in \mathcal{S}_{1} \cap \mathcal{S}_{2}$ to prove Theorem 1.3.

The above result guarantees the following compactness result for solution sequences to $(1)$ :

COROLlary 1.4. Let $\left\{\lambda_{1, n}\right\}$ and $\left\{\lambda_{2, n}\right\}$ be sequences of nonnegative constants satisfying (3) for some

$$
\left(\lambda_{1}, \lambda_{2}\right) \in\{[0,24 \pi) \backslash 8 \pi \mathbb{N}\} \times\{[0,24 \pi) \backslash 8 \pi \mathbb{N}\},
$$

and $\left\{v_{n}\right\} \subset E$ be a sequence of solutions to (1) corresponding to $\left(\lambda_{1, n}, \lambda_{2, n}\right)$. Then $\left\{v_{n}\right\}$ is relatively compact in $E$. 
The possible values of $\left(m_{1}\left(x_{0}\right), m_{2}\left(x_{0}\right)\right)$ for $x_{0} \in \mathcal{S}_{1} \cap \mathcal{S}_{2}$ will be more restrictive and we expect that

$$
\left(m_{1}\left(x_{0}\right), m_{2}\left(x_{0}\right)\right)=8 \pi\left(\frac{(\ell-1) \ell}{2}, \frac{\ell(\ell+1)}{2}\right), \quad 8 \pi\left(\frac{\ell(\ell+1)}{2}, \frac{(\ell-1) \ell}{2}\right)
$$

for $\ell=1,2,3, \cdots$, see [34]. To describe the background of this conjecture, let us define $\left(u_{1}, u_{2}\right) \in E \times E$ by

$$
u_{i}(x) \equiv \int_{M} G(x, y) \mu_{i}(y) d v_{g} \quad \text { for } i=1,2 .
$$

Then, the function $v=u_{1}-u_{2}$ satisfies (1). A basic idea is obtained by regarding these $u_{1}$ and $u_{2}$ as the positive and the negative parts of $v$, respectively, and in this case (1) becomes the Liouville system

$$
\begin{aligned}
& -\Delta_{g} u_{1}=\lambda_{1}\left(\frac{e^{a_{11} u_{1}+a_{12} u_{2}}}{\int_{M} e^{a_{11} u_{1}+a_{12} u_{2}} d v_{g}}-\frac{1}{|M|}\right), \\
& -\Delta_{g} u_{2}=\lambda_{2}\left(\frac{e^{a_{21} u_{1}+a_{22} u_{2}}}{\int_{M} e^{a_{21} u_{1}+a_{22} u_{2}} d v_{g}}-\frac{1}{|M|}\right), \\
& \int_{M} u_{1} d v_{g}=0, \quad \int_{M} u_{2} d v_{g}=0,
\end{aligned}
$$

with $a_{i j}(i, j=1,2)$ constituting

$$
A=\left(a_{i j}\right)=\left(\begin{array}{cc}
1 & -1 \\
-1 & 1
\end{array}\right)
$$

When this matrix is given by

$$
A=\left(\begin{array}{cc}
2 & -1 \\
-1 & 2
\end{array}\right)
$$

in (8), it comprises the $S U(3)$ Toda system (in the simplest form without the vortex term) arising in nonabelian relativistic self-dual gauge theory [22, 15, 41] studied by several authors mathematically $[18,19,26,7,32,17]$.

Each equation of the general Liouville system (8) is regarded as (4) by putting

$$
v=a_{i i} u_{i}, \quad \lambda=a_{i i} \lambda_{i}, \quad K=e^{a_{i j} u_{j}} \quad(j \neq i),
$$

for $i=1,2$ and, especially, to

$$
-\Delta_{g} v=\lambda\left(\frac{e^{v}}{\int_{M} e^{v} d v_{g}}-\frac{1}{|M|}\right), \quad \int_{M} v d v_{g}=0,
$$

if $\lambda_{1}$ or $\lambda_{2}=0$. Here, the equation (9) and its generalization (4) with the inhomogeneous coefficient $K(x)>0$ appear also in the self-dual gauge field theory [41], stationary system of chemotaxis or self-interacting particles [40], and the prescribing Gaussian curvature problem [1]. It has been studied in recent years $[29,39,4,24,23,5,6,21,38,14,36,31$, $33,2,13,8,9]$, and especially, we have the quantization phenomenon [23] of

$$
\lambda \in 8 \pi \mathbb{N}
$$

for the noncompact sequence of solutions $\left(v_{n}, \lambda_{n}\right)$ with $\lambda_{n} \rightarrow \lambda$ (based on [4, 24], see also $[29,39,36]$ for another method) and the classification of the singular limit using the Green 
function $[29,27,33]$. We note that these results are provided with fundamental tools or motivations for the variational method [38, 14], the singular perturbation of the solution (see [2] for bounded domain), and the calculation of the topological degree [23, 9].

Similar problems are also considered for $S U(3)$ Toda systems. Putting

$$
\mu_{1, n} \equiv \lambda_{1, n} \frac{e^{2 u_{1, n}-u_{2, n}}}{\int_{M} e^{2 u_{1, n}-u_{2, n}} d v_{g}}, \quad \mu_{2, n} \equiv \lambda_{2, n} \frac{e^{-u_{1, n}+2 u_{2, n}}}{\int_{M} e^{-u_{1, n}+2 u_{2, n}} d v_{g}},
$$

we obtain a result like the above Theorem $1.2([18,26,7])$. In this case, ( since $a_{i i}=2$ ) the estimate corresponding to $(5)$ is

$$
m_{i}\left(x_{0}\right) \geq 2 \pi \text { for each } x_{0} \in \mathcal{S}_{i} .
$$

Furthermore, if $x_{0} \in \mathcal{S}_{1} \cap \mathcal{S}_{2}$, then $m_{1}\left(x_{0}\right)$ and $m_{2}\left(x_{0}\right)$ satisfy the relation describing an ellipse

$$
m_{1}\left(x_{0}\right)^{2}-m_{1}\left(x_{0}\right) m_{2}\left(x_{0}\right)+m_{2}\left(x_{0}\right)^{2}=4 \pi\left(m_{1}\left(x_{0}\right)+m_{2}\left(x_{0}\right)\right),
$$

instead of (6). In fact, in the general form of (8), it holds that

$$
\begin{aligned}
& a_{11} a_{21} m_{1}\left(x_{0}\right)^{2}+2 a_{12} a_{21} m_{1}\left(x_{0}\right) m_{2}\left(x_{0}\right)+a_{22} a_{12} m_{2}\left(x_{0}\right)^{2} \\
& =8 \pi\left(a_{21} m_{1}\left(x_{0}\right)+a_{12} m_{2}\left(x_{0}\right)\right) .
\end{aligned}
$$

For the $S U(3)$ Toda case, the improvement of the estimate (10) to

$$
m_{i}\left(x_{0}\right) \geq 4 \pi \text { for each } x_{0} \in \mathcal{S}_{i}
$$

was obtained in [26], see also [32, 17]. In this case it is expected that

$$
\left(m_{1}\left(x_{0}\right), m_{2}\left(x_{0}\right)\right) \in\{(4 \pi, 8 \pi),(8 \pi, 4 \pi),(8 \pi, 8 \pi)\}
$$

for any $x_{0} \in \mathcal{S}_{1} \cap \mathcal{S}_{2}$.

2. Preliminaries. In this section, we describe several results obtained in [34] to be used in the proof of the main theorem of this paper.

First, given $x_{0} \in \mathcal{S}_{1} \cap \mathcal{S}_{2}$, we take an isothermal chart $(\Psi, U)$ satisfying

$$
\Psi\left(x_{0}\right)=0, \quad \Psi(x)=X \in \mathbb{R}^{2}, \quad g=e^{\xi(X)}\left(d X_{1}^{2}+d X_{2}^{2}\right),
$$

and $\bar{U} \cap\left(\mathcal{S}_{1} \cup \mathcal{S}_{2}\right)=\left\{x_{0}\right\}$. Then, $v_{n}(X)=v_{n} \circ \Psi^{-1}(X)$ is a solution of

$$
-\Delta v_{n}=\lambda_{1, n}\left(\frac{e^{v_{n}}}{\int_{M} e^{v_{n}}}-\frac{1}{|M|}\right) e^{\xi}-\lambda_{2, n}\left(\frac{e^{-v_{n}}}{\int_{M} e^{-v_{n}}}-\frac{1}{|M|}\right) e^{\xi} \quad \text { in } \Omega .
$$

Let us define the functions $h_{\xi}$ by

$$
\Delta h_{\xi}=e^{\xi} \quad \text { in } \Omega, \quad h_{\xi}=0 \quad \text { on } \partial \Omega,
$$

where $\Omega=\Psi(U) \subset \mathbb{R}^{2}$. Without loss of generality, we may assume that $\partial \Omega$ is smooth.

Putting

$$
\begin{aligned}
& w_{1, n}(X)=v_{n}\left(\Phi^{-1}(X)\right)-\log \int_{M} e^{v_{n}}-\frac{\lambda_{1, n}-\lambda_{2, n}}{|M|} h_{\xi}, \\
& w_{2, n}(X)=-v_{n}\left(\Phi^{-1}(X)\right)-\log \int_{M} e^{-v_{n}}-\frac{-\lambda_{1, n}+\lambda_{2, n}}{|M|} h_{\xi},
\end{aligned}
$$


we obtain

$$
\begin{aligned}
& -\Delta w_{1, n}=V_{1, n}(x) e^{w_{1, n}}-V_{2, n} e^{w_{2, n}}, \\
& -\Delta w_{2, n}=-V_{1, n}(x) e^{w_{1, n}}+V_{2, n}(x) e^{w_{2, n}},
\end{aligned}
$$

in $\Omega$ for

$$
\begin{aligned}
& V_{1, n}=\lambda_{1, n} e^{\xi+\frac{\lambda_{1, n}-\lambda_{2, n}}{|M|} h_{\xi}}, \\
& V_{2, n}=\lambda_{2, n} e^{\xi+\frac{-\lambda_{1, n}+\lambda_{2, n}}{|M|} h_{\xi}},
\end{aligned}
$$

satisfying

$$
\begin{aligned}
& 0 \leq V_{1, n}(X) \leq b, \quad 0 \leq V_{2, n}(X) \leq b \quad(\forall X \in \Omega) \\
& \int_{\Omega} e^{w_{1, n}} \leq c, \quad \int_{\Omega} e^{w_{2, n}} \leq c,
\end{aligned}
$$

with some constants $b, c>0$ independent of $n$, and

$$
\begin{aligned}
& V_{1, n} \quad \rightarrow \quad V_{1}=\lambda_{1} e^{\xi+\left(\lambda_{1}-\lambda_{2}\right) h_{\xi}}, \\
& V_{2, n} \quad \rightarrow \quad V_{2}=\lambda_{2} e^{\xi+\left(-\lambda_{1}+\lambda_{2}\right) h_{\xi}},
\end{aligned}
$$

uniformly on $\bar{\Omega}$. By (5) we have only to consider the case $\min \left(\lambda_{1}, \lambda_{2}\right)>0$, that is, $V_{1}, V_{2}>0$. We have $x_{i, n} \rightarrow x_{0}$ such that $v_{n}\left(x_{1, n}\right),-v_{n}\left(x_{2, n}\right) \rightarrow+\infty$. This implies $X_{i, n}=\Psi\left(x_{i, n}\right) \rightarrow 0$ and also

$$
v_{n}\left(x_{n}\right)-\log \int_{M} e^{v_{n}}, \quad-v_{n}\left(x_{n}\right)-\log \int_{M} e^{-v_{n}} \rightarrow+\infty
$$

from the proof of [34, Lemma 2.2], or equivalently, $w_{i, n} \rightarrow+\infty$ for each $i=1,2$. This means $0 \in \mathcal{S}_{i}^{0}$, where

$$
\mathcal{S}_{i}^{0}=\left\{X_{0} \in \Omega \mid \text { there exists } X_{n} \rightarrow X_{0} \text { such that } w_{i, n}\left(X_{n}\right) \rightarrow+\infty\right\} .
$$

We also obtain $\mathcal{S}_{i}^{0}=\Psi\left(U \cap \mathcal{S}_{i}\right)=\{0\}$ similarly to the proof of [34, Lemma 2.2].

Next, by Theorem 1.2 we have

$$
\begin{array}{lll}
V_{1, n} e^{w_{1, n}} & \rightarrow & m_{1} \delta_{0}+r_{1}, \\
V_{2, n} e^{w_{2, n}} & \rightarrow & m_{2} \delta_{0}+r_{2},
\end{array}
$$

in $\mathcal{M}(\bar{\Omega})$ with $\min \left(m_{1}, m_{2}\right) \geq 4 \pi, r_{1}, r_{2} \in L^{1}(\Omega) \cap L_{l o c}^{\infty}(\bar{\Omega} \backslash\{0\})$, and

$$
V_{i, n} e^{w_{i, n}} \rightarrow r_{i} \quad \text { in } L_{l o c}^{p}(\bar{\Omega} \backslash\{0\})
$$

for any $1 \leq p<\infty$. These $m_{i}$ coincide with $m_{i}\left(x_{0}\right)(i=1,2)$. By Theorem 1.2 we have $r_{1}=0$ and $r_{2}=0$ in the cases of $m_{1}-m_{2}>4 \pi$ and $-m_{1}+m_{2}>4 \pi$, respectively, and

$$
\left(m_{1}-m_{2}\right)^{2}=8 \pi\left(m_{1}+m_{2}\right) \text {. }
$$

Thus, we obtain (11), (12), and (13) in a bounded domain $\Omega \subset \mathbb{R}^{2}$, taking $x=\left(x_{1}, x_{2}\right)$ to indicate the standard coordinates in $\mathbb{R}^{2}$. We have to show $m_{i} \geq 8 \pi(i=1,2)$ to prove the main theorem. Here, we recall that Brezis-Merle [4] type theorem for (11) holds by a similar argument discussed for the $S U(3)$ Toda system [26, Theorem 4.2].

LEMma 2.1. If $\left\{\left(w_{1, n}, w_{2, n}\right)\right\}_{n}$ is a solution sequence to (11) and (12), then there is a subsequence (denoted by the same symbol) satisfying the following alternatives. 
(1) Both $\left\{w_{1, n}\right\}_{n}$ and $\left\{w_{2, n}\right\}_{n}$ are locally uniformly bounded in $\Omega$.

(2) There is $i \in\{1,2\}$ such that $\left\{w_{i, n}\right\}_{n}$ is uniformly bounded in $\Omega$ and $w_{j, n} \rightarrow-\infty$ locally uniformly in $\Omega$ for $j \neq i$.

(3) Both $w_{1, n} \rightarrow-\infty$ and $w_{2, n} \rightarrow-\infty$ locally uniformly in $\Omega$.

(4) For the blow-up sets $\mathcal{S}_{1}^{0}, \mathcal{S}_{2}^{0}$ defined for this subsequence, we have $\mathcal{S}_{1}^{0} \cup \mathcal{S}_{2}^{0} \neq \emptyset$ and $\sharp\left(\mathcal{S}_{1}^{0} \cup \mathcal{S}_{2}^{0}\right)<+\infty$. Furthermore, for each $i \in\{1,2\}$, either $\left\{w_{i, n}\right\}_{n}$ is locally uniformly bounded in $\Omega \backslash\left(\mathcal{S}_{1}^{0} \cup \mathcal{S}_{2}^{0}\right)$ or $w_{i, n} \rightarrow-\infty$ locally uniformly in $\Omega \backslash\left(\mathcal{S}_{1}^{0} \cup \mathcal{S}_{2}^{0}\right)$. Here, if $\mathcal{S}_{i}^{0} \backslash\left(\mathcal{S}_{1}^{0} \cap \mathcal{S}_{2}^{0}\right) \neq \emptyset$, then $w_{i, n} \rightarrow-\infty$ locally uniformly in $\Omega \backslash\left(\mathcal{S}_{1}^{0} \cup \mathcal{S}_{2}^{0}\right)$, and each $x_{0} \in \mathcal{S}_{i}^{0}$ takes $m_{i}\left(x_{0}\right) \geq 4 \pi$ such that

$$
V_{i, n}(x) e^{w_{i, n}} \rightarrow \sum_{x_{0} \in \mathcal{S}_{i}^{0}} m_{i}\left(x_{0}\right) \delta_{x_{0}} \quad \text { *-weakly in } \mathcal{M}(\Omega) .
$$

Finally, performing the rescaling argument using the above lemma, we arrive at one of the following:

(1) (Liouville equation in $\mathbb{R}^{2}$ )

$$
-\Delta w=e^{w} \quad \text { in } \mathbb{R}^{2}, \quad \int_{\mathbb{R}^{2}} e^{w}<+\infty,
$$

(2) (singular Liouville equation in $\mathbb{R}^{2}$ )

$$
-\Delta w=e^{w}-\sum_{x_{0} \in \mathcal{S}} m\left(x_{0}\right) \delta_{x_{0}}, \quad \int_{\mathbb{R}^{2}} e^{w}<+\infty,
$$

where $\mathcal{S} \subset \mathbb{R}^{2}$ is a finite set and $m\left(x_{0}\right) \geq 4 \pi$ for any $x_{0} \in \mathcal{S}$.

LEMMA 2.2 ([10, 11]). We have the following:

(1) For the solution $w$ to (14) we have $\int_{\mathbb{R}^{2}} e^{w}=8 \pi$.

(2) For the solution $w$ to (15) we have $\int_{\mathbb{R}^{2}} e^{w}>4 \pi+\sum_{x_{0} \in \mathcal{S}} m\left(x_{0}\right)$.

REMARK 2.3. Lemma 2.2 (2) follows from [11, Theorem 2.3], but the statement there assumes $0>m\left(x_{0}\right)>-4 \pi$ for each $x_{0} \in \mathcal{S}$, which seems not to cover our cases $m\left(x_{0}\right) \geq$ $4 \pi$. Nevertheless Lemma 2.2 (2) holds because the proof of [11, Theorem 2.3] is applicable to our cases. Indeed, it is necessary to show

$$
v(x):=w(x)+\frac{m\left(x_{0}\right)}{2 \pi} \log \left|x-x_{0}\right|^{-1} \leqq C+C_{1} \log (|x|+1)
$$

for some constants $C$ and $C_{1}$ in each sufficiently small neighbourhood of $x_{0} \in \mathcal{S}$, say $B_{\varepsilon}\left(x_{0}\right)$, in the course of the proof. Here we note that $v(x)$ satisfies

$$
-\Delta v=V(x) e^{v} \quad \text { in } B_{\varepsilon}\left(x_{0}\right)
$$

with

$$
V(x)=e^{-\frac{m\left(x_{0}\right)}{2 \pi} \log \left|x-x_{0}\right|^{-1}}=\left|x-x_{0}\right|^{\frac{m\left(x_{0}\right)}{2 \pi}},
$$

which belongs to $L^{\infty}\left(B_{\varepsilon}\left(x_{0}\right)\right)$ if $m\left(x_{0}\right) \geq 0$ (and to $L^{p}\left(B_{\varepsilon}\left(x_{0}\right)\right)$ for some $p \in(1, \infty)$ if $\left.0>m\left(x_{0}\right)>-4 \pi\right)$. Now taking smaller $\varepsilon>0$ if necessary, we get (16) with $C_{1}=0$ from $[4$, Corollary 4]. 
3. Proof of Theorem 1.3. We have $\mathcal{S}_{1}^{0}=\mathcal{S}_{2}^{0}=\{0\}$, and there are $x_{1, n} \rightarrow 0$ and $x_{2, n} \rightarrow 0$ such that

$$
w_{1, n}\left(x_{1, n}\right)=\sup _{\Omega} w_{1, n} \rightarrow+\infty \quad \text { and } \quad w_{2, n}\left(x_{2, n}\right)=\sup _{\Omega} w_{2, n} \rightarrow+\infty .
$$

We take the rescaling of $w_{i, n}$ around $x_{k, n}$ by

$$
w_{i, n}^{k}(x)=w_{i, n}\left(x_{k, n}+\varepsilon_{k, n} x\right)-w_{k, n}\left(x_{k, n}\right),
$$

where $i, k=1,2$ and $\varepsilon_{k, n}=e^{-w_{k, n}\left(x_{k, n}\right) / 2}$. Then

$$
\begin{aligned}
& -\Delta w_{1, n}^{k}=V_{1, n}\left(x_{k, n}+\varepsilon_{k, n} x\right) e^{w_{1, n}^{k}}-V_{2, n}\left(x_{k, n}+\varepsilon_{k, n} x\right) e^{w_{2, n}^{k}}, \\
& -\Delta w_{2, n}^{k}=-V_{1, n}\left(x_{k, n}+\varepsilon_{k, n} x\right) e^{w_{1, n}^{k}}+V_{2, n}\left(x_{k, n}+\varepsilon_{k, n} x\right) e^{w_{2, n}^{k}}
\end{aligned}
$$

in $\Omega_{n}^{k}=\left\{x \in \mathbb{R}^{2} \mid \frac{x-x_{k, n}}{\varepsilon_{k, n}} \in \Omega\right\}$ with $\int_{\Omega_{i, n}^{k}} e^{w_{i, n}^{k}}=\int_{\Omega} e^{w_{i, n}} \leq b$. Without loss of generality, we may suppose

$$
\varepsilon_{1, n} \leq \varepsilon_{2, n}
$$

for $n=1,2, \cdots$, i.e., $w_{1, n}\left(x_{1, n}\right) \geq w_{2, n}\left(x_{2, n}\right)$. Then, we take the rescaled solution around $x_{1, n}$, i.e., $\left(w_{1, n}^{1}, w_{2, n}^{1}\right)$. Since

$$
\begin{aligned}
& w_{1, n}^{1}(x) \leq w_{1, n}^{1}(0)=0 \\
& w_{2, n}^{1}(x) \leq w_{2, n}^{1}\left(\frac{x_{2, n}-x_{1, n}}{\varepsilon_{1, n}}\right) \leq w_{2, n}\left(x_{2, n}\right)-w_{1, n}\left(x_{1, n}\right) \leq 0
\end{aligned}
$$

on $\Omega_{n}^{1}$, Lemma 2.1 assures the following alternatives:

(1) Both $\left\{w_{1, n}^{1}\right\}$ and $\left\{w_{2, n}^{1}\right\}$ are locally uniformly bounded in $\mathbb{R}^{2}$.

(2) $\left\{w_{1, n}^{1}\right\}$ is locally uniformly bounded in $\mathbb{R}^{2}$, while $w_{2, n}^{1} \rightarrow-\infty$ locally uniformly in $\mathbb{R}^{2}$.

The first alternative, however, never occurs. Indeed, we have

$$
\begin{aligned}
w_{2, n}^{1}(x)= & w_{2, n}\left(x_{1, n}+\varepsilon_{1, n} x\right)-w_{1, n}\left(x_{1, n}\right) \\
= & w_{1, n}\left(x_{1, n}+\varepsilon_{1, n} x\right)+w_{2, n}\left(x_{1, n}+\varepsilon_{1, n} x\right) \\
& \quad-\left(w_{1, n}\left(x_{1, n}+\varepsilon_{1, n} x\right)-w_{1, n}\left(x_{1, n}\right)\right)-2 w_{1, n}\left(x_{1, n}\right) \\
= & w_{1, n}\left(x_{1, n}+\varepsilon_{1, n} x\right)+w_{2, n}\left(x_{1, n}+\varepsilon_{1, n} x\right)-w_{1, n}^{1}(x)-2 w_{1, n}\left(x_{1, n}\right)
\end{aligned}
$$

and, from the definition of $w_{i, n}$, we have also

$$
w_{1, n}(x)+w_{2, n}(x)=-\log \int_{M} e^{v_{n}}-\log \int_{M} e^{-v_{n}} .
$$

Here it follows from the Jensen inequality that

$$
\log \int_{M} e^{v_{n}} \geq \log |M|, \quad \log \int_{M} e^{-v_{n}} \geq \log |M|
$$

and consequently we have

$$
w_{1, n}(x)+w_{2, n}(x) \leq-2 \log |M| .
$$

From these we obtain

$$
w_{2, n}^{1}(x) \leq-2 \log |M|-w_{1, n}^{1}(x)-2 w_{1, n}\left(x_{1, n}\right) \rightarrow-\infty
$$

for every $x \in \mathbb{R}^{2}$ if $\left\{w_{1, n}^{1}(x)\right\}$ is locally uniformly bounded in $\mathbb{R}^{2}$. 
Henceforth, we consider the second alternative concerning this rescaling around $x_{1, n}$. Then we have a subsequence (denoted by the same symbol) such that $w_{1, n}^{1} \rightarrow w_{1}^{1}$ in $C_{l o c}^{1, \alpha}\left(\mathbb{R}^{2}\right)$ and this $w_{1}^{1}$ satisfies

$$
-\Delta w_{1}^{1}=V_{1}(0) e^{w_{1}^{1}}, \quad \int_{\mathbb{R}^{2}} e^{w_{1}^{1}}<+\infty .
$$

Therefore, from the first case of Lemma 2.2 we have

$$
m_{1} \geq \int_{\mathbb{R}^{2}} V_{1}(0) e^{w_{1}^{1}}=8 \pi .
$$

Henceforth, we put $w_{2}^{1}=-\infty$ for simplicity, and therefore, this alternative is referred to as $w_{1}^{1} \in C_{\text {loc }}^{1, \alpha}\left(\mathbb{R}^{2}\right)$ and $w_{2}^{1}=-\infty$. Furthermore, we have $\left(m_{1}, m_{2}\right) \geq(8 \pi, 4 \pi)$, namely, $m_{1} \geq 8 \pi$ and $m_{2} \geq 4 \pi$.

Now, we use the rescaled solution $\left(w_{1, n}^{2}, w_{2, n}^{2}\right)$ around $x_{2, n}$. In this case, we have

$$
\begin{aligned}
& w_{2, n}^{2}(x) \leq w_{2, n}^{2}(0)=0 \\
& w_{1, n}^{2}(x) \leq w_{1, n}^{2}\left(\frac{x_{1, n}-x_{2, n}}{\varepsilon_{2, n}}\right)=w_{1, n}\left(x_{1, n}\right)-w_{2, n}\left(x_{2, n}\right),
\end{aligned}
$$

in $\Omega_{n}^{2}$. In spite of $w_{1, n}\left(x_{1, n}\right)-w_{2, n}\left(x_{2, n}\right) \geq 0$, again by Lemma 2.1 we have the following alternatives.

(1) Both $\left\{w_{1, n}^{2}\right\}$ and $\left\{w_{2, n}^{2}\right\}$ are locally uniformly bounded in $\mathbb{R}^{2}$.

(2) $\left\{w_{2, n}^{2}\right\}$ is locally uniformly bounded, while $w_{1, n}^{2} \rightarrow-\infty$ locally uniformly in $\mathbb{R}^{2}$.

(3) There is a finite blow-up set $\mathcal{S}_{1}^{2}$ of $\left\{w_{1, n}^{2}\right\}$ such that $V_{1, n}\left(x_{2, n}+\varepsilon_{2, n} x\right) e^{w_{1, n}^{2}} \rightarrow$ $\sum_{x_{0} \in \mathcal{S}_{1}^{2}} m_{1}^{2}\left(x_{0}\right) \delta_{x_{0}}$ in $\mathcal{M}\left(\mathbb{R}^{2}\right)$ with $m_{1}^{2}\left(x_{0}\right) \geq 4 \pi$ for any $x_{0} \in \mathcal{S}_{1}^{2}$ and $w_{1, n}^{2} \rightarrow-\infty$ locally uniformly in $\mathbb{R}^{2} \backslash \mathcal{S}_{1}^{2}$. Moreover, either

3 -i) $\left\{w_{2, n}^{2}\right\}$ is locally uniformly bounded in $\mathbb{R}^{2} \backslash \mathcal{S}_{1}^{2}$, or

3-ii) $w_{2, n}^{2} \rightarrow-\infty$ locally uniformly in $\mathbb{R}^{2} \backslash \mathcal{S}_{1}^{2}$.

Here the first alternative is impossible by the preceding argument of the rescaling around $x_{1, n}$ and we proceed to the other cases.

The second alternative is indicated by $w_{2}^{2} \in C_{l o c}^{1, \alpha}\left(\mathbb{R}^{2}\right)$ and $w_{1}^{2}=-\infty$. The former function satisfies the Liouville equation on $\mathbb{R}^{2}$, and this implies $m_{2} \geq 8 \pi$. On the other hand, we have already $m_{1} \geq 8 \pi$ from the former rescaling. Therefore, $\left(m_{1}, m_{2}\right) \geq(8 \pi, 8 \pi)$.

In the first case of the third alternative, passing to a subsequence, we have $w_{2, n}^{2} \rightarrow w_{2}^{2}$ in $C_{l o c}^{1, \alpha}\left(\mathbb{R}^{2} \backslash \mathcal{S}_{1}^{2}\right)$ with $w_{2}^{2}$ satisfying

$$
-\Delta w_{2}^{2}=V_{2}(0) e^{w_{2}^{2}} \quad \text { in } \mathbb{R}^{2} \backslash \mathcal{S}_{1}^{2} .
$$

Here we note that $w_{2}^{2} \leq 0$ since $w_{2, n}^{2}(x) \leq 0$, which guarantees $\int_{\mathbb{R}^{2}} e^{w_{2}^{2}}<\infty$. Moreover, it follows that there exist $\varphi \in L_{\text {loc }}^{1}\left(\mathbb{R}^{2}\right)$ and constants $\alpha\left(x_{0}\right)>0$ for each $x_{0} \in \mathcal{S}_{1}^{2}$ such that

$$
-\Delta w_{2}^{2}=-\sum_{x_{0} \in \mathcal{S}_{1}^{2}} \alpha\left(x_{0}\right) \delta_{x_{0}}+\varphi \quad \text { in } \mathbb{R}^{2},
$$


see [3, Theorem 1]. It is easy to see that $\varphi=V_{2}(0) e^{w_{2}^{2}}$ and $\alpha\left(x_{0}\right)=m_{1}^{2}\left(x_{0}\right)$. Consequently $w_{2}^{2}$ satisfies the following

$$
\begin{aligned}
& -\Delta w_{2}^{2}=-\sum_{x_{0} \in \mathcal{S}_{1}^{2}} m_{1}^{2}\left(x_{0}\right) \delta_{x_{0}}+V_{2}(0) e^{w_{2}^{2}} \quad \text { in } \mathbb{R}^{2}, \\
& \int_{\mathbb{R}^{2}} e^{w_{2}^{2}}<+\infty
\end{aligned}
$$

where $m_{1}^{2}\left(x_{0}\right) \geq 4 \pi$ for each $x_{0} \in \mathcal{S}_{1}^{2}$. In particular,

$$
\int_{\mathbb{R}^{2}} V_{2}(0) e^{w_{2}^{2}}>4 \pi+\sum_{x_{0} \in \mathcal{S}_{1}^{2}} m_{1}^{2}\left(x_{0}\right)
$$

by the second case of Lemma 2.2, and therefore,

$$
m_{2}>4 \pi+\sum_{x_{0} \in \mathcal{S}_{1}^{2}} m_{1}^{2}\left(x_{0}\right)>8 \pi .
$$

Finally, the second case of the third alternative does not occur. In fact, we have $w_{2, n}^{2}(0)=0$, and therefore, $0 \in \mathcal{S}_{1}^{2}$. We can choose $R>0$ satisfying $\overline{B_{R}(0)} \cap \mathcal{S}_{1}^{2}=\{0\}$, and define $h_{i, n}(i=1,2)$ by

$$
\begin{array}{ll}
-\Delta h_{i, n}=V_{i, n}\left(x_{2, n}+\varepsilon_{2, n} x\right) e^{w_{i, n}^{2}} & \text { in } B_{R}(0), \\
h_{i, n}=0 \quad \text { on } \partial B_{R}(0) . &
\end{array}
$$

Then,

$$
h_{0, n}=w_{2, n}^{2}-\left(h_{2, n}-h_{1, n}\right)
$$

is a harmonic function satisfying

$$
\sup _{B_{R}(0)} h_{0, n} \leq \sup _{\partial B_{R}(0)} h_{0, n} \rightarrow-\infty .
$$

On the other hand, we have $0 \leq e^{w_{2, n}^{2}(x)} \leq e^{0}=1$ and $e^{w_{2, n}^{2}}(x) \rightarrow 0$ locally uniformly in $\mathbb{R}^{2} \backslash \mathcal{S}_{1}^{2}$, and therefore, $e^{w_{2, n}^{2}(x)} \rightarrow 0$ in $L^{p}\left(B_{R}(0)\right)$ for every $p \in[1, \infty)$. This implies

$$
h_{2, n} \rightarrow 0 \quad \text { in } C^{1, \alpha}\left(B_{R}(0)\right),
$$

while $h_{1, n}$ is a nonnegative function. Thus, we obtain

$$
\begin{aligned}
& 0=w_{2, n}^{2}(0)=h_{0, n}(0)+h_{2, n}(0)-h_{1, n}(0) \leq h_{0, n}(0)+h_{2, n}(0) \\
& \leq \sup _{B_{R}(0)} h_{0, n}+\left\|h_{2, n}\right\|_{L^{\infty}\left(B_{R}(0)\right)} \rightarrow-\infty
\end{aligned}
$$

a contradiction, and the proof is complete.

\section{References}

[1] T. Aubin, Some Nonlinear Problems in Riemannian Geometry, Springer, Berlin, 1998.

[2] S. Baraket and F. Pacard, Construction of singular limits for a semilinear elliptic equation in dimension 2, Calc. Var. Partial Differential Equations 6 (1998), 1-38.

[3] H. Brezis and P. L. Lions, A note on isolated singularities for linear elliptic equations, in: Mathematical Analysis and Applications, Part A, Adv. in Math. Suppl. Stud. 7A (1981), 263-266. 
[4] H. Brezis and F. Merle, Uniform estimates and blow-up behavior for solutions of $-\Delta u=$ $V(x) e^{u}$ in two dimensions, Comm. Partial Differential Equations 16 (1991), 1223-1253.

[5] E. Caglioti, P. L. Lions, C. Marchioro, and M. Pulvirenti, A special class of stationary flows for two-dimensional Euler equations: A statistical mechanics description, Comm. Math. Phys. 143 (1992), 501-525.

[6] E. Caglioti, P. L. Lions, C. Marchioro, and M. Pulvirenti, A special class of stationary flows for two-dimensional Euler equations: A statistical mechanics description, part II, Comm. Math. Phys. 174 (1995), 229-260.

[7] D. Chae, H. Ohtsuka, and T. Suzuki, Some existence results for SU(3) Toda system, Calc. Var. Partial Differential Equations 17 (2005), 235-255.

[8] C.-C. Chen and C.-S. Lin, Sharp estimates for solutions of multi-bubbles in compact Riemannian surfaces, Comm. Pure Appl. Math. 55 (2002), 728-771.

[9] C.-C. Chen and C.-S. Lin, Topological degree for a mean field equations on Riemann surfaces, Comm. Pure Appl. Math. 56 (2003), 1667-1803.

[10] W. Chen and C. Li, Classification of solutions of some nonlinear elliptic equations, Duke Math. J. 63 (1991), 615-622.

[11] W. Chen and C. Li, What kinds of singular surfaces can admit constant curvature?, Duke Math. J. 78 (1995), 437-451.

[12] A. J. Chorin, Vorticity and Turbulence, Springer, New York, 1994.

[13] M. del Pino and J. Wei, Collapsing steady states of the Keller-Segel system, Nonlinearity 19 (2006), 661-684.

[14] W. Ding, J. Jost, J. Li, and G. Wang, Existence results for mean field equations, Ann. Inst. H. Poincaré Anal. Non Linéaire 16 (1999), 653-666.

[15] G. Dunne, Self-dual Chern-Simons Theories, Lecture Notes in Phys. 36, Springer, Berlin, 1995.

[16] L. Fontana, Sharp borderline Sobolev inequalities on compact Riemannian manifolds, Comment. Math. Helv. 68 (1993), 415-454.

[17] J. Jost, C. S. Lin, and G. Wang, Analytic aspects of the Toda system: II. Bubbling behavior and existence of solutions, Comm. Pure Appl. Math. 59 (2006), 526-558.

[18] J. Jost and G. Wang, Analytic aspects of the Toda system: I. A Moser-Trudinger inequality, Comm. Pure Appl. Math. 54 (2001), 1289-1319.

[19] J. Jost and G. Wang, Classification of solutions of a Toda system in $\mathbb{R}^{2}$, International Mathematical Research Notices 6 (2002), 277-290.

[20] G. Joyce and D. Montgomery, Negative temperature states for the two-dimensional guiding-centre plasma, J. Plasma Phys. 10 (1973), 107-121.

[21] M. K. H. Kiessling, Statistical mechanics of classical particles with logarithmic interactions, Comm. Pure Appl. Math. 46 (1993), 27-56.

[22] C. Kim, C. Lee, P. Ko, B.-H. Lee, and H. Min, Schrödinger fields on the plane with $[U(1)]^{N}$ Chern-Simons interactions and generalized self-dual solitons, Physical Review D 48 (1993), 1822-1840.

[23] Y. Y. Li, Harnack type inequality: the method of moving planes, Comm. Math. Phys. 200 (1999), 421-444.

[24] Y. Y. Li and I. Shafrir, Blow-up analysis for solutions of $-\Delta u=V e^{u}$ in dimension two, Indiana Univ. Math. J. 43 (1994), 1255-1270.

[25] P. L. Lions, On Euler Equations and Statistical Physics, Scuola Normale Superiore, Pisa, 1997. 
[26] M. Lucia and M. Nolasco, SU(3) Chern-Simons vortex theory and Toda systems, J. Differential Equations 184 (2002), 443-474.

[27] L. Ma and J. C. Wei, Convergence for a Liouville equation, Comment. Math. Helv. 76 (2001), 506-514.

[28] C. Marchioro and M. Pulvirenti, Mathematical Theory of Incompressible Nonviscous Fluids, Springer, New York, 1994.

[29] K. Nagasaki and T. Suzuki, Asymptotic analysis for two-dimensional elliptic eigenvalue problems with exponentially-dominated nonlinearities, Asymptotic Analysis 3 (1990), 173188.

[30] P. K. Newton, The N-Vortex Problem: Analytical Techniques, Springer, New York, 2001.

[31] H. Ohtsuka and T. Suzuki, Palais-Smale sequence relative to the Trudinger-Moser inequality, Calc. Var. Partial Differential Equations 17 (2003), 235-255.

[32] H. Ohtsuka and T. Suzuki, Blowup analysis for SU(3) Toda system, RIMS Kokyuroku 1405 (2004), 73-99.

[33] H. Ohtsuka and T. Suzuki, Blow-up analysis for Liouville type equation in self-dual gauge field theories, Comm. Contemp. Math. 7 (2005), 177-205.

[34] H. Ohtsuka and T. Suzuki, Mean field equation for the equilibrium turbulence and a related functional inequality, Adv. Differential Equations 11 (2006), 281-304.

[35] Y. B. Pointin and T. S. Lundgren, Statistical mechanics of two-dimensional vortices in a bounded container, Phys. Fluids 19 (1976), 1459-1470.

[36] T. Senba and T. Suzuki, Some structures of the solution set for a stationary system of chemotaxis, Adv. Math. Sci. Appl. 10 (2000), 191-224.

[37] I. Shafrir and G. Wolansky, Moser-Trudinger and logarithmic HLS inequalities for systems, J. Eur. Math. Soc. 7 (2005), 413-448.

[38] M. Struwe and G. Tarantello, On multivortex solutions in Chern-Simons gauge theory, Boll. Unione Mat. Ital. Sez. B Artic. Ric. Mat. (8) 1 (1998), 109-121.

[39] T. Suzuki, Semilinear Elliptic Equations, Gakkotosho, Tokyo, 1994.

[40] T. Suzuki, Free Energy and Self-Interacting Particles, Birkhäuser, Boston, 2005.

[41] Y. Yang, Solitons in Field Theory and Nonlinear Analysis, Springer, New York, 2001. 
